\title{
IMPLICACIONES ÉTICAS, LEGALES, RELIGIOSAS Y SOCIOECONÓMICAS DE LA FECUNDACIÓN IN VITRO EN LA SALUD REPRODUCTIVA DE COSTA RICA ${ }^{1}$
}

Institución: Posgrado en Enfermería Ginecológica, Obstétrica y Perinatal de la Universidad de Costa Rica

\section{COMO CITAR}

Campos,G. (2012).Implicaciones éticas, legales, religiosas y socioeconómicas de la fecundación in vitro en la Salud Reproductiva de Costa Rica. [En línea].Rev. Enfermería Actual en Costa Rica, 22, 1-14 [citado (fecha)]. Disponible World Wide Web: <http://www.revenf.ucr.ac.cr/vitro.pdf> ISSN $1409-4568$

\section{RESUMEN}

Las técnicas emergentes de reproducción humana asistida, entre las que se encuentran la fecundación in vitro (FIV), suscitan una serie de interrogantes científicas, éticas y sociales. En relación con el tema, Costa Rica ha generado controversia a nivel mundial, pues no ha legalizado dicha técnica de fecundación, ya que parte de argumentos jurídicos que, según la OPS y la $\mathrm{CIDH}$, van en contra de los derechos humanos y reproductivos. Por la situación anterior es que a continuación se describen las implicaciones éticas, legales, religiosas y socioeconómicas de la fecundación in vitro en la salud reproductiva de Costa Rica. Se realizó una revisión y análisis documental. Se buscó información en las siguientes bases de datos: PubMed, Medline, Excelencia.net, EBSCO, Cochrane. Se encontraron referentes bibliográficos que enfocan los aspectos religiosos, legales, éticos y socioeconómicos, los cuales se analizaron a la luz de los derechos reproductivos y la implicación de esta técnica en la salud reproductiva de Costa Rica. La posición de los y las Enfermeras y el mundo científico, así como las decisiones tomadas por usuarios, deben estar acompañadas de una información amplia y correcta acerca de los métodos, de las oportunidades de embarazo y de los efectos secundarios que la FIV pueda acarrear, de modo que contemple tanto cuidados preconcepcionales, prenatales y perinatales, de allí que el y la enfermera obstetra tiene un papel importante en la atención integral de la mujer durante el período prenatal, el parto y el postparto, así como de la asistencia inmediata y eficiente del recién nacido. Se concluye que la fecundación in vitro constituye una técnica médica que se utiliza en muchas partes del mundo para tratar la esterilidad. Puede beneficiar a los usuarios individuales y a la sociedad en general, no sólo porque trata la esterilidad, sino porque también ofrece la posibilidad de evitar los defectos genéticos y mejora la investigación sobre la reproducción y la anticoncepción humanas; sin embargo, ésta debe ser accesible a toda la población, de manera que realmente se garanticen los derechos humanos, pero debe ser regulada estrictamente, de manera que se respete la vida humana en lugar de comerciarla.

Palabras clave: Costa-Rica, derechos-reproductivos, fecundación-in-vitro

\footnotetext{
${ }^{1}$ Fecha de recepción: 22 de noviembre 2012

Fecha de aceptación: 29 de febrero 2012

${ }^{2}$ Enfermera. Estudiante de la Maestría en Enfermería Ginecológica, Obstétrica y Perinatal de la Universidad de Costa Rica. Correo electrónico: grettelcamposv@gmail.com
} 


\title{
ETHICAL, LEGAL, RELIGIOUS AND SOCIOECONOMIC IMPLICATION BY VITRO FERTILIZATION IN REPRODUCTIVE HEALTH IN COSTA RICA ${ }^{1}$
}

Grettel Campos Vargas ${ }^{2}$

Institution: University of Costa Rica. Postgraduate degree in Nursing Gynecology, Obstetric and Perinatal

\section{CITED}

Campos,G. (2012).Ethical, legal, religious and socioeconomic implication by vitro fertilization in Reproductive Health in Costa Rica. [Electronic version].Rev. Enfermería Actual en Costa Rica, 22, 1-14 [cited (date)]. Available World Wide Web: <http://www.revenf.ucr.ac.cr/vitro.pdf> ISSN $1409-4568$

\begin{abstract}
The emerging techniques of assisted human reproduction, including those found in vitro fertilization (IVF), raise a number of scientific questions, especially ethical and social. Costa Rica has attracted controversy worldwide, it has not legalized such fertilization technique because of legal arguments, according to PAHO and the Commission, are against human and reproductive rights. A review and document analysis information was sought on the following databases: PubMed, Medline, Excelencia.net, EBSCO, Cochrane. Bibliographic references were found and analyzed on the basis of reproductive rights and ownership of this technique in the reproductive health of Costa Rica. The position of nurses, the scientific world and the decisions made by users should be accompanied by a comprehensive and accurate information about methods, the chances of pregnancy and side effects of IVF can carry so much care in the period of preconception, prenatal and perinatal. Hence the professional Nursing Obstetric and Perinatal has an important role in the comprehensive care of women during the prenatal, delivery and postpartum, as well as immediate care and efficient assistance the newborn. It is concluded that IVF is a medical technique used in many parts of the world to treat infertility. It can benefit individual users and society in general, not only because it is infertility, but also because it offers the possibility to avoid genetic defects and improving research on human reproduction and contraception, it must be accessible the entire population, so that really guarantee human rights, must be strictly regulated so that human life is respected rather than trade it.
\end{abstract}

Keywords: Costa Rica, reproductive-rights, in-vitro-fertilization.

\footnotetext{
${ }^{1}$ Date of reception: November 22, 2012

Date of acceptance: February 29, 2012

${ }^{2}$ Enfermera. Estudiante de la Maestría en Enfermería Ginecológica, Obstétrica y Perinatal de la Universidad de Costa Rica. E mail: grettelcamposv@gmail.com
} 


\section{INTRODUCCION}

En 1995, el Ministerio de Salud de Costa Rica emitió el decreto ejecutivo 24029-S1 denominado 'Fertilización in vitro y transferencia de embriones' (Bustos, 2007), el cual aprobaba la reproducción asistida homóloga entre cónyuges, aunque incluía varios requisitos, entre ellos que se tratara del último medio técnico para conseguir el embarazo y que, habiendo sido informados acerca de la adopción, hayan renunciado a ella. Además, permitía la reproducción asistida heteróloga con otros requisitos (Escobar, 2007)

Posteriormente, la Sala Constitucional de la Corte Suprema de Justicia costarricense, por sentencia del 15 de marzo de 2002, con el voto disidente de dos magistrados, declaró inconstitucional el decreto 24029-S1 por razones de fondo y forma (Arguedas, 2007). Entre de los argumentos principales expuestos se menciona que a partir de la fecundación ya existe persona, además de que no hay que pasar por alto que el embrión es un sujeto de derecho y no un mero objeto; respecto de dicha postura, el artículo 4.1 del Pacto de San José dispone el respeto a la vida desde la concepción, de allí que las Técnicas de Reproducción Asistida (TRA) representan un atentado contra la vida humana, debido a la pérdida elevada de embriones, considerados seres humanos.

En junio del 2011, Costa Rica incumplió, por tercera vez, el plazo dado por la Comisión Interamericana de Derechos Humanos (CIDH) para aprobar la fecundación in vitro, pues el Congreso archivó un proyecto de ley en la materia, lo que expuso al país a una demanda internacional.

Con una votación de 26 contra 25, los diputados desecharon el proyecto de ley con el que el Gobierno pretendía regular esta técnica de reproducción asistida para evitar que el país sea acusado ante la Corte Interamericana de Derechos Humanos (Corte IDH), cuya sede se encuentra precisamente en San José.

La técnica in vitro, que permite la fecundación fuera del cuerpo de la madre, está prohibida en el país desde el 2000 y enfrenta una férrea oposición de parte de entidades religiosas.

Las polémicas sobre su legalización han generado una fuerte polémica en el congreso costarricense desde octubre del 2010, luego de que la CIDH llamara la atención al país por violentar los derechos humanos de las parejas que no pueden tener hijos.

El primer plazo dado por la CIDH a Costa Rica para legalizar la técnica de FIV venció en febrero del 2011. El actual gobierno costarricense pidió una primera prórroga, hasta el 31 de mayo, y una segunda, que venció el 31 de julio del 2011. Actualmente, el país enfrenta una demanda por la CIDH, ante la negativa de legalizar la fecundación in vitro en el país.

Dado que este tratamiento brinda a muchas parejas la oportunidad de procrear, es imperante que los gobernantes analicen y reflexionen en torno a dicha temática.

Aunado a lo anterior, el profesional en Enfermería Obstétrica tiene un papel relevante, ya que es la persona a la que acude la pareja cuando quiere aclarar dudas sobre procedimientos o tratamientos o para buscar apoyo y consejo en distintas áreas, particularmente durante la consejería en salud sexual y salud reproductiva. 
Durante los procesos de salud-enfermedad, el profesional en Enfermería es uno de los profesionales que se encarga de guiar y explicar todo a los usuarios al brindarles información correcta, actualizada y basada en la mejor evidencia, así como al organizar las pruebas necesarias y pertinentes relacionadas con la infertilidad y la administración del tratamiento. De igual manera, junto con otros profesionales, comparten la responsabilidad de organizar el trabajo, así como de atender el aspecto psicológico de los pacientes, ya que la esterilidad/infertilidad conlleva problemas biológicos, psicológicos y sociales tales como el sentimiento de culpa por diversos factores; no obstante, al determinarse la causa, es posible que uno de los miembros se autoinculpe, máxime que la infertilidad se considera un tabú, razón por la cual suele ser ocultada por quien la padece, al punto de que la pareja se excluye de la vida social y ese aislamiento produce una carga emocional que amenaza la armonía y la estabilidad de ambos. (Colomer, 2001)

Las anteriores son algunas razones por las cuales los profesionales en Enfermería, en particular los especializados en el área ginecoobstétrica, deben manifiestarse en relación con la problemática costarricense referente a la FIV.

El objetivo de esta revisión es brindar un panorama del contexto actual de Costa Rica relacionado con la fecundación in vitro desde una perspectiva ética, legal, religiosa, socioeconómica y la perspectiva desde Enfermería Gineco-obstétrica.

\section{MATERIALES Y METODOS}

Se revisaron las bases de datos de la Biblioteca de Ciencias de la Salud de la Universidad de Costa Rica, en donde se exploraron libros de Embriología humana, Enfermería Gineco-obstétrica, Bioética y Técnicas en reproducción asistida, así como artículos de revistas científicas almacedados en bases de datos como Imbiomed, Scielo, CORIS, Bioethikos y documentos publicados por la Universidad Nacional de México (UNAM), la Organización Panamericana de la Salud (OPS) y la Organización Mundial de la Salud (OMS); los cuales sirvieron como referentes de la temática en estudio.

También se valoró la literatura no técnica, entre la que se encuentra los Informes de la Sentencia del Tribunal Constitucional de Costa Rica, los cuales prohiben la fecundación in vitro en dicho país y que igualmente contienen refutaciones a esta práctica; también se incluyeron videos de noticieros de Costa Rica relativos a la opinión de los magistrados y algunos diputados de la Asamblea Legislativa de Costa Rica con referencia a la FIV; se revisó periódicos como La Nación, La República y Al Día, documentos como la Sentencia del Tribunal Constitucional de Costa Rica (2000), La Gaceta (2003); Decreto Ejecutivo; Nuevas formas de dominación y desigualdad: las tecnologías de reproducción asistida y el cuerpo femenino (Arguedas, sf), Fecundación in vitro una discusión nacional ( Arguedas y Calzada 2005), entre otros.

Los datos recolectados se analizaron de acuerdo con los factores éticos, legales, religiosos y socioeconómicos propuestos en esta revisión a la luz del contexto nacional. 


\section{RESULTADOS}

\section{Factores éticos y legales}

La Técnica de Fecundación In Vitro, en los términos en que se regula en Costa Rica con el Decreto Ejecutivo $\mathrm{N}^{\circ}$ 24029-S, no es incompatible con el derecho a la vida ni a la dignidad humana, sino que constituye un instrumento que la ciencia y la técnica han concedido al ser humano para favorecerla, ya que la infertilidad es vista como consecuencia de un estado de enfermedad, por lo que debe ser atendida dentro de este contexto, ya sea con fines preventivos, diagnósticos y terapéuticos. (Arguedas, 2005)

Por otra parte, la Sala Constitucional no cierra por completo la posibilidad de aceptar la FIV, siempre que esta demuestre no atentar contra la vida según los avances tecnológicos. Así se manifiesta:

Según la Sala ha podido constatar, la aplicación de la Técnica de Fecundación In Vitro y Transferencia Embrionaria, en la forma en que se desarrolla en la actualidad, atenta contra la vida humana. Este Tribunal sabe que los avances de la ciencia y la biotecnología son tan vertiginosos que la técnica podría llegar a ser mejorada de tal manera, que los reparos señalados aquí desaparezcan. Sin embargo, las condiciones en las que se aplica actualmente, llevan a concluir que cualquier eliminación o destrucción de concebidos -voluntaria o derivada de la impericia de quien ejecuta la técnica o de la inexactitud de ésta- viola su derecho a la vida, por lo que la Técnica no es acorde con el Derecho de la Constitución y por ello el reglamento cuestionado es inconstitucional por infracción al artículo 21 de la Constitución Política y 4 de la Convención Americana sobre Derechos Humanos. (Sala constitucional de la suprema corte de justicia, 2000, p. 13)

De acuerdo con la literatura consultada, se demuestra que constantemente la técnica se está mejorando para el bien de la humanidad y a favor de la vida, es aquí donde tanto los estudios clínicos y científicos basados en la mejor evidencia, así como los profesionales en salud involucrados en el área juegan un papel determinante. No obstante, en Costa Rica no se cuenta con estudios científicos actualizados al respecto que fundamenten realmente la validez o no de la FIV.

En cuanto a la perspectiva ética, Roca (1998) considera que los principios que se derivan de la protección de la dignidad humana y que son aplicables a las técnicas de FIV, por ejemplo el derecho a heredar un patrimonio genético no manipulado y el derecho a que las TRA, sean empleadas exclusivamente con finalidad terapéutica.

Asimismo Más, Gonzalez y Cobas (2009) determinan que:

Al revisar las regulaciones y disposiciones vigentes en diversos países, así como las recomendaciones de comités y organismos internacionales, se comprueba que existen aspectos comunes en lo que respecta a la utilización de las técnicas actuales. Estos son:

- El acceso a éstas técnicas debe estar limitado a parejas heterosexuales casadas legalmente, o que mantengan una unión estable.

- Las instituciones y el personal médico que ofrecen estos servicios deben estar sujetos a supervisión y regulación sanitaria. 
- La paternidad y la maternidad deben estar determinadas por leyes que rijan para todos los nacimientos logrados mediante estas técnicas.

- Las historias clínicas y la información deben conservarse con carácter confidencial.

- La vida embrionaria in vitro debe estar limitada a 14 días.

- El almacenamiento de gametos y embriones debe estar limitado en tiempo.

- La implantación y/o la inseminación postmorten debe estar prohibida.

- Las agencias o intermediarios para la subrogación deben estar prohibidas.

- Previamente debe obtenerse el consentimiento de los participantes.

- Las tecnologías reproductivas deben estar libres de comercialización.

- No debe haber selección del sexo, excepto en caso de enfermedades hereditarias ligadas al sexo, ni tampoco selección eugenésica.

- Prohibición absoluta de la manipulación genética por técnicas extremas de ingeniería genética (clonaje, creación de quimeras partenogénesis, fertilización entre especies y otras).” (2009, p.7-8)

Para ello se determinan varios principios, independientemente de las variaciones políticas, culturales y religiosas, los cuales se resumen a: el respeto por la dignidad humana, la seguridad del material genético, la calidad de los servicios brindados, la inviolabilidad de la persona, la inalienabilidad del cuerpo humano.

Los tres primeros constituyen mecanismos de protección de la persona y los restantes son un medio para asegurar el control sobre la libertad personal y científica, así como el respeto a la dignidad humana que es en sí el origen de todos los derechos humanos fundamentales. (Más, Gonzalez y Cobas, 2009)

\section{Factores religiosos}

Respecto de los factores religiosos, dos de las instancias morales que se han pronunciado sobre el reconocimiento de la dignidad humana del embrión desde el momento de la fecundación y que han exigido el respeto sobre su patrimonio cromosómico y genético son la Declaración de la Iglesia Evangélica de Alemania sobre las Cuestiones de Bioética y la Instrucción Donum Vitae o la Carta Encíclica "Evangelium Vitae". Tales instancias defienden la vida humana desde el momento de la concepción, al igual que la constitución política de Costa Rica, las cuales se apegan a las instituciones religiosas del país, ya que tienen gran influencia política y social.

Asimismo, el 31 de noviembre de 1993, en la Plaza de San Pedro el Papa Juan Pablo II criticó los experimentos científicos que están orientados a manipular la vida humana, respecto de lo que se mostró profundamente preocupado sobre las investigaciones ilícitas e inquietantes que violan las normas éticas y menosprecian la dignidad humana alegando que "muchas cosas cambian en el hombre y su entorno, pero su naturaleza no puede ser alterada".(p.74)

No obstante, por ello, el Papa exhortó a los científicos a que protejan al embrión humano de los experimentos genéticos o de lo contrario correrán el riesgo de "poner a la humanidad en peligro" (p.74), y de ahí que increpara a los gobiernos para que no se descuiden ante los avances de la información genética. 


\section{Factores socioeconómicos}

En cuanto a los factores socioeconómicos, la prohibición de la FIV en Costa Rica no implica que las mujeres costarricenses no puedan someterse a ese procedimiento, sino que este no se practica en territorio nacional. Una consecuencia de esta situación es que cada día más mujeres optan por salir del país para acceder a este tipo de tratamiento, tal como lo describe Arguedas (sf), los lugares que comúnmente visitan son Panamá y Colombia, traslado que implica un aumento en los costos de quienes desean optar por la maternidad bajo esta técnica, además, el acceso a la información y los cuidados suele ser menores, situación que muchas veces desemboca en partos múltiples atendidos en los hospitales del sistema nacional de salud pública que, suelen presentar muchas complicaciones de las cuales las pacientes no están ni parcialmente enteradas, escenario injusto tanto para las madres como para el sistema de salud pública. (Arguedas, s.f.)

Las mujeres que desean y pueden pagar la FIV, al salir del país para aplicárselo en algún otro lugar, quedan en muchos casos, en estado de indefensión legal, máxime que se están enfrentando a un mercado de características tan delicadas, rodeado de tabúes, sensibilidades y particularidades culturales, la ausencia de regulación puede desencadenar enormes diferencias en precios, accesibilidad, situación de los oferentes y demandantes y en el marco jurídico en que estas interacciones se den; claro está, que estas condiciones favorecen la desigualdad y explotación de las mujeres que participen a lo largo de las distintas etapas en estos procesos, proceso que se torna aún más complejo cuando hay compra de óvulos y alquiler de úteros. Esta ambigüedad legal sirve a la industria que lucra exitosamente, pero sin que rinda cuentas claras al punto de que, inclusive, ni siquiera hay estadísticas confiables acerca de la tasa de éxito, los casos fatales, las consecuencias de las tecnologías y las limitaciones. (Arguedas, s.f.)

Ante tal panorama, el profesional en Enfermería Obstétrica y Perinatal debe estar actualizado e informado en relación con la FIV en el contexto costarricense y pronunciarse al respecto, ya que juega un papel importante al ser uno de los entes de salud responsable de la atención integral de la mujer durante el período prenatal, el parto y el postparto, así como de brindar una asistencia inmediata y eficiente al recién nacido, acciones que constituyen las tres intervenciones más importantes para consolidar una buena salud perinatal, lo cual es exigido por la Caja Costarricense de Seguro Social (CCSS) en la guía de atención integral a las mujeres niños y niñas en el periodo prenatal, parto y posparto del 2008.

\section{DISCUSION}

Actualmente un tema de gran controversia en Costa Rica en salud reproductiva es la Fecundación in vitro como una TRA que involucra la fecundación extracorpórea.

Cabe destacar que según la OMS, las Técnicas de Reproducción Asistida corresponden a todos los tratamientos o procedimientos que incluyen la manipulación tanto de ovocitos como de espermatozoides o embriones humanos para el establecimiento de un embarazo. Aunque no se limita a la FIV, sí la incluye al igual que otras prácticas como la transferencia de embriones, la transferencia intratubárica de gametos, la transferencia intratubárica de cigotos, la transferencia intratubárica de embriones, la criopreservación de ovocitos y embriones, la donación de ovocitos y embriones y el útero surrogado, mas no incluye la inseminación asistida (inseminación artificial) usando espermatozoides ni de la pareja ni de un donante. (OMS, 2010). Teniendo en cuenta lo 
mencionado, el primer argumento de Costa Rica se ciñe al concepto de persona jurídica, término del Derecho, pero que contiene una base teológica personalista. (Arguedas, 2007.) La segunda tesis considera que el embrión es sujeto de derecho y no un mero objeto, ya que se respeta la vida desde el momento de la fecundación, idea que incluye los principios de la Bioética.

Para la tercera manifestación, hay que recordar que la Convención Americana sobre Derechos Humanos, conocida como 'Pacto de San José de Costa Rica', suscrita en la Conferencia Especializada Interamericana sobre Derechos Humanos, fue llevada a cabo precisamente en San José, Costa Rica, entre el 7 y el 22 de noviembre de 1969. Se adoptó este último día y entró en vigor el 18 de julio de 1978, conforme al Artículo 74.2 de la Convención. Su depositario es la Secretaría General OEA. Al respecto, en el Capítulo II se refiere a los derechos civiles y políticos y su Artículo 4 trata sobre el derecho a la vida, cuya redacción inicia así: "1. Toda persona tiene derecho a que se respete su vida. Este derecho estará protegido por la ley y, en general, a partir del momento de la concepción. Nadie puede ser privado de la vida arbitrariamente"(p.70). Aquí el problema más serio se relaciona con la confusión terminológica respecto de la inconstitucionalidad del decreto 24029-S1. El detalle procede del sentido de la palabra concebir, concepto que alude a la concepción vista como el inicio del embarazo.

Por otra parte, la OMS y todas las sociedades científicas de la Ginecología y la Obstetricia del mundo, así como las de reproducción asistida, aceptan que el embarazo inicia con la implantación del embrión, no con la fecundación; dichas entidades, además, toman fecundación (unión de los gametos) e implantación como sinónimos, aunque fecundación es la unión de un ovocito con un espermatozoide y la combinación de sus materiales genéticos que resulta posteriormente en la formación de un cigoto, mientras que la implantación corresponde a la unión y la subsecuente penetración del blastocisto libre de zona pelúcida, usualmente en el endometrio, que comienza 5 a 7 días después de la fecundación. Desde aquí se puede observar que corresponden a dos procesos consecutivos pero distintos.

Finalmente, el cuarto y último argumento es la pérdida elevada de embriones, cuyo análisis se realiza dudando de la eficacia de la fecundación in vitro, máxime teniendo en cuenta que los datos utilizados son de casi una década atrás. Cabe destacar que la eficacia ha mejorado tanto en este momento, que el argumento mencionado sería fácil de debatir, ya que, en muchos casos, aplicar una TRA es casi tan eficaz como lograr un embarazo con una fecundación no asistida, esto es, sin tratamiento médico.

Asimismo, la experiencia de Costa Rica, según lo discutido por Álvarez (2007), deja ver la influencia de argumentos religiosos en relación con los biojurídicos, cuyas discrepancias han impedido establecer un adecuado diálogo entre ambas.

A partir del marco referencial costarricense, la mayor divergencia resulta de la consideración del inicio de la vida o embarazo, ya que para Costa Rica este empieza con un óvulo fecundado, pero, médicamente se considera que una mujer está embarazada cuando un óvulo fecundado se implanta en el recubrimiento de su útero y sólo entonces puede desarrollarse como un feto. La aseveración anterior se fundamenta tomando en cuenta que según la OMS se declara el estado de embarazo una vez que el huevo fecundado se implanta en el útero materno. Este fenómeno ocurre dentro de los primeros diez días después de producida la fecundación. (OMS, 2005). 
De esta manera parecería resuelto el dilema, ya que la OMS es la máxima autoridad en salud a nivel internacional; sin embargo, al revisar la literatura utilizada para la formación de profesionales en salud se encuentran las siguientes definiciones:

"El desarrollo de un individuo comienza con la fecundación, fenómeno por el cual el espermatozoide del varón y el ovocito de la mujer se unen para dar origen a un nuevo organismo, el cigoto". (Langman, 2001, p. 3)

"El desarrollo humano es un proceso continuo que se inicia cuando un oocito de una mujer es fecundado por un espermatozoo del varón”.(Moore, 2007, p. 1)

"La fecundación es el fenómeno biológico mediante el cual se unen un espermatozoide y un óvulo para formar una nueva célula-denominada huevo o cigoto-, con la que se inicia el periodo embrionario, es decir, la vida de un nuevo individuo" (Hib, 1999, p. 8)

Con este panorama queda claro que al analizar la fecundación in vitro no solo se debe tomar en cuenta la definición de embarazo o inicio de la vida humana, tal como se definen anteriormente, ya que a pesar de que los libros de texto para la formación de profesionales en salud la conciben de una manera, para la OMS es definida de otra forma, por lo tanto, se requiere de un espectro más amplio de análisis donde se evalúe no solo la definición, forma y contenido, sino que se consideren determinantes sociales, derechos humanos, bioética, tecnología y desarrollo.

Por otra parte, se puede utilizar la actualización de Obstetricia y Ginecología del 2010, en la que Urries, al analizar la situación referente a la elección del embrión óptimo, de alta capacidad implantatoria, concluye que por ahora posiblemente no haya ningún procedimiento para seleccionar un embrión totalmente válido, pero si se concretaran las expectativas puestas en la metabolómica, la cual permite seleccionar al mejor embrión, con mayor potencial de implantación, mediante un procedimiento no invasivo, su combinación con los criterios de selección morfológicos podrían suponer un avance muy importante en la búsqueda del embrión óptimo, máxime que estas técnicas "no invasivas" son de aplicación sencilla, rápidas, de bajo costo monetario y reproducibles. (Urries, 2010)

De esta manera es indispensable evocar los derechos reproductivos que abarcan determinados derechos humanos reconocidos por las leyes nacionales e internacionales. Estos derechos se basan en el reconocimiento básico del derecho de todas las parejas y personas a decidir con libertad y responsabilidad el número de hijos que quieren tener, con qué frecuencia y en qué momento y a disponer de la información y los medios para lograrlo; también, comprenden su derecho a tomar decisiones acerca de la reproducción sin que haya discriminación, coacción o violencia, de modo que las parejas infértiles pueden recibir un diagnóstico, así como prevenir la infertilidad secundaria, sin olvidar que la FIV ofrece la posibilidad de evitar los defectos genéticos y mejorar la investigación básica sobre la reproducción y la anticoncepción humanas.

En ocasiones resulta difícil identificar la causa de infertilidad de una pareja, ya que esta puede deberse a secuelas de las infecciones de transmisión sexual, del aparato reproductor y de enfermedades generalizadas como la tuberculosis, el paludismo o la infección por el VIH (OMS, 2000). Igualmente, hay que considerar aquellas 
causas que imposibilitan finalizar un embarazo, entre las que se encuentran las infecciones de transmisión sexual como la sífilis, las complicaciones de partos o abortos peligrosos previos, los tumores y otros muchos factores.

Asimismo, la infertilidad masculina, cuya causa inmediata es un semen de mala calidad o escaso, puede ser consecuencia de una obstrucción de los conductos deferentes o de un trastorno de la producción de espermatozoides, originados a su vez por determinadas infecciones de transmisión sexual, por algunas enfermedades generales o por factores hormonales, ambientales o de hábitos de vida como el tabaquismo o el consumo excesivo de alcohol y drogas (De Francisco, 2007)

Ante el panorama descrito, se destacan las Técnicas de Reproducción Asistida, una amplia gama de procedimientos que tienen como finalidad aumentar las posibilidades de concepción a través de un acercamiento por diversos medios- entre óvulo y espermatozoide, las cuales se ofrecen como un medio para ejercer el legítimo ejercicio del derecho a la reproducción humana que, aunque no está expresamente reconocido en nuestra Constitución Política, se deriva del derecho a la libertad y la autodeterminación, el derecho a la intimidad personal y familiar y la libertad para fundar una familia. El derecho a la reproducción involucra, el propio derecho a la vida, ya no en su dimensión individual, sino en una colectiva: el derecho a contribuir a la preservación y continuidad de la especie humana.

Apegándose al derecho reproductivo, las condiciones antes citadas deben tratarse médicamente, para ello existen tratamientos contra la infertilidad que comprenden múltiples estrategias médicas y extramédicas, aunque en su mayor parte no están al alcance de la mayoría de las parejas de los países en vías de desarrollo, como Costa Rica.

De hecho, podría decirse que los adelantos realizados en el campo de las técnicas de reproducción asistida, como la fecundación in vitro, la transferencia intratubárica de gametos o cigotos, la inyección intracitoplásmica de espermatozoides y la congelación de embriones, representan un caso clásico de desigualdad entre las inversiones y las necesidades, ya que al ser ilegal en Costa Rica solo las parejas que puedan pagar el tratamiento privado en el extranjero y viajar tienen acceso al procedimiento. (Datta, 2002). Lo anterior, además, deja ver debilidad económica en el sistema de salud del seguro social, entidad que debe encargarse de tratar infecciones de transmisión sexual y el suministro de medios para curar dichas infecciones y la enfermedad inflamatoria pélvica, como los antibióticos; el tratamiento quirúrgico de las obstrucciones orgánicas femeninas y masculinas; y las estrategias extramédicas como el asesoramiento acerca de la frecuencia y el momento óptimos para el coito y otras modificaciones de la conducta. (De francisco, 2007)

Asimismo, los estudios sociales, clínicos y operativos ayudarían a identificar las intervenciones esenciales en los niveles personal, de pareja y social, para prevenir las enfermedades frecuentes que provocan infertilidad, así como para diagnosticar y tratar la esterilidad con métodos sencillos, cuando sea posible; para reducir el estigma de la esterilidad mediante el asesoramiento de la pareja y de la familia; para mejorar la educación de la sociedad y el apoyo a los acuerdos alternativos para el intercambio de niños, como la acogida o la adopción, entre otras opciones.(OMS, 2007)

Por otra parte, la ética especializada se encarga de evaluar la FIV para Costa Rica: en este caso, la Bioética estudia, en forma interdisciplinaria, los problemas creados a raíz del proceso científico técnico en los campos 


\section{Revista Electrónica Enfermería Actual en costa Rica}

médico y biológico, tanto a nivel microsocial como macrosocial y su repercusión en la sociedad y sus sistemas sociales. (Blázquez, 2008). Cuando las cuestiones afectan a grupos sociales y no solamente a personas en situación individual y las decisiones pueden afectar y aun negar intereses individuales en procura del bien de la comunidad, se acude a la Bioética.

En este sentido es importante tener en cuenta el peligro implícito en equiparar lo ético con lo legal, porque siempre es posible que una ley vigente permita (es decir, considere legal) algo que no sea aceptable en las normas de conducta de un grupo humano en un momento o que, por el contrario, prohíba (tomándolo ilegal) algo que el grupo acepta como conducta de sus miembros y que se ajusta al respeto por la condición humana, el caso de la fecundación in vitro ante la controversia que genera su ilegalidad en Costa Rica.

El tema de la Bioética no es un campo lejano en la práctica clínica cotidiana de los y las profesionales en salud. En la historia reciente, se ha mencionado que la industrialización de la medicina ha producido una proletarización del trabajo médico, debido en parte al desarrollo acelerado de la tecnología médica diagnóstica y terapéutica, campos que constantemente se enfrentan ante dilemas morales.

Además, el respeto a la propia dignidad que tiene cada ser humano obliga a toda persona a actuar siempre de acuerdo con su propia posición, una vez adoptada libre y razonadamente, además de que ningún individuo en su sano juicio podría respetar y acatar prácticas e ideas que en no respete en otros contextos.

Costa Rica debe dedicar esfuerzos a un análisis científico, social, económico, ético y jurídico que garantice la práctica regulada de la FIV o la desapruebe como lo hace actualmente, pero para ello debe contar con bases teóricas probadas y fundamentadas, en lugar de dedicarse solamente a pedir prórrogas, cuando el espectro de la temática es mucho más amplio.

Ante este panorama religioso, histórico, social, económico, jurídico y ético de la FIV en Costa Rica como derecho reproductivo, es claro que la temática tiene muchas aristas de análisis y no solo la validez de los derechos humanos y reproductivos como apelan muchas personalidades nacionales y entidades internacionales, por ende, se requiere de un exhaustivo estudio de la situación del país frente a la temática, su regulación, su implementación y constante actualización.

Debido a la difusión de los avances en Obstetricia como el procedimiento metabolómico y de los diferentes movimientos mundiales para humanizar el nacimiento, estudios han demostrado seguras y eficaces prácticas clínicas y cuidados basados en la mejor evidencia científica disponible excluyendo todas aquellas utilizadas de forma rutinaria que producen mayores riesgos y complicaciones para las madres y los recién nacidos.

Por lo tanto es pertinente contextualizar la atención integral en salud para las familias que optan por la FIV, ya sea que se realice dentro o fuera del país, brindándoles los cuidados con enfoque de riesgo desde la atención primaria centrada en las mujeres y sus bebés. En este sentido, el enfoque de atención de las mujeres, los niños y niñas, así como de sus familias en los diferentes aspectos de la salud reproductiva y en particular, durante el embarazo y nacimiento, requiere de una transformación indispensable basada en estudios científicos que contemplen un análisis de los determinantes sociales en salud, las condiciones y la forma en que las mujeres viven su embarazo y parto, ya que tienen impacto en sus vidas y la de sus hijas(os), por lo que los procesos de atención 
en el enfoque mencionado se deben sustentar en evidencia y no en mitos o costumbres que favorecen la implantación de prácticas nocivas en la atención de las madres y sus bebés.

Se requiere, entonces, estudio y actualización continua del profesional en Enfermería Ginecológica, Obstétrica y Perinatal en TRA y, particularmente, en la FIV, pues, como se ha discutido, a pesar de que no se realice en el país, la atención de las mujeres que se someten a este tratamiento fuera de Costa Rica vuelven para recibir los cuidados prenatales, del parto y posparto.

\section{CONCLUSIONES}

- La fecundación in vitro y el trasplante de embriones constituyen una técnica médica que se utiliza en muchas partes del mundo para tratar la esterilidad. Puede beneficiar a los usuarios individuales y a la sociedad en general, no sólo porque trata la esterilidad, sino porque también ofrece la posibilidad de evitar los defectos genéticos y mejorar la investigación básica sobre la reproducción y la anticoncepción humanas; sin embargo, debe ser accesible a toda la población de manera que realmente se garanticen los derechos humanos, pues debe garantizarse el respeto por la vida y no la comercialización de esta.

- El lucro con la infertilidad, así como el riesgo que las nuevas técnicas pueden causar cuando son empleadas por personal no calificado, han obligado a establecer e imponer cierto tipo de control, tanto sobre los que la practican, como sobre los beneficiarios, ya que toda práctica clínica debe estar sujeta a legislaciones en materia de salud o a códigos de conducta deontológica, por ende, aquellos centros o profesionales que practiquen las técnicas de la concepción asistida deben estar sujetos a regulaciones específicas y provistos de la acreditación adecuada.

- Independientemente de cualquier consideración legal, hay consenso general de que el embrión humano merece el reconocimiento inherente a su dignidad como miembro de la familia humana. Sólo así Costa Rica puede proceder a examinar qué prácticas son contrarias al respeto de esa dignidad, de modo que pueda brindar protección al material genético humano.

- Las autoridades religiosas reconocen la dignidad humana del embrión desde la fecundación y exigen respeto a la vida y al patrimonio genético.

- En nuestra sociedad democrática, la mujer y la familia no pueden ser discriminadas en su deseo de reproducción. Los cambios en la estructura familiar, la división del trabajo, el lugar que ocupa y que está ocupando en la sociedad, traen nuevos desafíos, por lo cual es necesario establecer límites respecto de los usos de las FIV. Sin embargo, estos límites no deben transformarse en limitaciones con un carácter prohibicionista, los cuales desarrollan una dinámica en la cual pueden insertarse solo aquellos con elevados medios económicos que les permita trasladarse al extranjero para solucionar su problemática, brecha que crea una desigualdad de oportunidades que empaña leyes realmente necesarias. 
- Mediante el desarrollo de estructuras de encuentro permanente de información y de consenso, cuyo objetivo sea tener un control sobre los aspectos éticos en la utilización de las TRA, los profesionales en Enfermería y el mundo científico, propician que las decisiones que los usuarios tomen estén acompañadas de información amplia, y correcta de los métodos, así como de las oportunidades de embarazo y de los efectos secundarios que la FIV pueda acarrear en lo que respecta a cuidados preconcepcionales, prenatales y perinatales.

\section{REFERENCIAS}

Alvarez, J. (2007) El estatus del embrión humano desde el gradualismo. Gac Med Mex. Imbiomed. México. 2007; 143(3):267-77. Recuperado de

http://www.imbiomed.com.mx/1/1/articulos.php?method=showDetail\&id_articulo=45411\&id_seccion=1899\&id ejemplar= $\underline{4597 \& \mathrm{id} \text { revista }=48}$

Alvarez, J. (2008) El estatus biológico del embrión humano. El quimerismo interespecies como nuevo problema bioético y biojurídico. Madrid, España: Aranzadi.

Alvarez, J. (2009) Sobre la posibilidad de la donación de embriones en Hispanoamérica. An Fac med. 2009 ; $70(2): 1354$. Recuperado de http://www.scielo.org.pe/pdf/afm/v70n2/a09v70n2.pdf

Arguedas, C., y Calzada, A. (2007) Refutación de las objeciones de dos de los magistrados a la Sentencia del Tribunal Constitucional de Costa Rica prohibiendo la fecundación in vitro en dicho país. Vida Humana Internacional. Miami, USA. Recuperado de http://www.vidahumana.org/vidafam/repro/refutacion.html

Arguedas C., Calzada A. (2007) Sentencia del Tribunal Constitucional de Costa Rica prohibiendo la fecundación in vitro en dicho país. Vida Humana Internacional Miami, USA. Recuperado de http://www.vidahumana.org/vidafam/repro/costaricainvitro.html

Arguedas, C., Calzada, A. (2005). Fecundación in vitro una discusión nacional. CORIS. Vol. 1. Numero 3. ITCR. Costa Rica. Recuperado de http://www.tec.cr/sitios/docencia/cienciassociales/revista_coris/anteriores/CORIS.pdf

Arguedas, G. (s.f.) Nuevas formas de dominación y desigualdad: las tecnologías de reproducción asistida y el cuerpo femenino. Universidad de Costa Rica. San José. Recuperado de

http://congresoderechosreproductivos.com/files/docscongreso/repasistida/DominacionyTecnologia.pdf

Blazquez, N., García, L. (2008) Bioética fundamental. Madrid, España: (s.e.)

Bustos, D. (2007) Evolución de la bioética en Costa Rica: una historia reciente._Centro Universitário São Camilo. 2007; 1(2):39-44. Recuperado de http://www.saocamilosp.br/pdf/bioethikos/57/Evolucion_de_la bioetica_en_Costa_Rica.pdf

Caja Costarricense de Seguro Social (2008) Guía de Atención Integral a las Mujeres, Niños y Niñas en el periodo prenatal, parto y posparto. San José, Costa Rica: CCSS.

Colomer, D. (2001) Enfermería maternal y ginecológica. Barcelona, España: Masson. 


\section{Revista Electrónica Enfermería Actual en costa Rica}

Congregación para la Doctrina de la Fe. (1988) Instructio de observantia erga vitam human amnascentem de que procreation is dignitatetuenda. Acta Apostolicae Sedis; 80, 70-102.

Datta y OMS. (2002) Current practices and controversies in assisted reproduction: report of a WHO meeting. Ginebra, Suiza: OMS

De Francisco, A; Dixón, R; Aranguez, C. (2007) Aspectos de la investigación sobre la salud sexual y reproductiva en países con ingresos bajos e intermedios. Foro mundial para la investigación Sobre la salud. OMS. ISBN 2-940286-50-7. Recuperado de http://whqlibdoc.who.int/publications/2007/2940286507 spa.pdf

Escobar, I. (2007) Derecho a la reproducción humana (inseminación y fecundación in vitro). Cuestiones Constitucionales. Revista mexicana de derecho constitucional. UNAM. 2007; 16 (1), 137-58. Recuperado de http://www.juridicas.unam.mx/publica/rev/cconst/cont/16/ard/ard5.htm

Hib, J. (1999) Embriología Médica. México D.F., México: McGraw-Hill Interamericana.

Iglesia Evangélica de Alemania. (1986) Declaración sobre bioética.La Documentation Catholique; 83, 325-328.

Juan Pablo II. (1995) Carta encíclica Evangelium Vitae de 25 de marzo de 1995. Acta Apostolicae Sedis; 87, 401-522.

La Gaceta. (2003) Decreto Ejecutivo n.31078. Imprenta Nacional; mar 27, (61). San José.

Langman., J. (2001) Embriología Médica. México D. F., México: Ed. Panamericana - Williams Wilkins.

Más, J., González, J., Cobas, M., González, P., Pérez, A. (2007) Aspectos éticos y legales de la reproducción asistida. Extraído desde:http://www.crmmt.cfm.org.br/biblioteca_virtual/des_etic/16.htm

Moore, K; Persaud, T; Torchia, M. (2007) Embriología clínica. México D. F., México: Mc Graw-Hill.

OMS e ICMART. (2010) Glosario de terminologías en técnicas de reproducción asistida. Recuperado de http://www.who.int/reproductivehealth/publications/infertility/art_terminology_es.pdf

OMS. (2000) WHO manual for the investigation, diagnosis and management of the infertile male. Ginebra, Suiza: OMS.

OMS (1993) WHO manual for the standardized investigation and diagnosis of the infertile couple. Ginebra, Suiza: OMS.

OPS y OMS. (2005) Programa mujer y desarrollo. Anticoncepción de emergencia en las Américas. Recuperado de http://www.paho.org/spanish/ad/ge/emergencycontraceptionsp.PDF

Roca, E. (1998) Derechos de reproducción y eugenesia en biotecnología y derecho. Perspectivas en derecho comparado. Publicaciones de la cátedra de derecho y genoma humano. Granada, España: Ed. Comares Bilbao.

Sala constitucional de la suprema corte de justicia. (2000) Sentencia del Tribunal Constitucional de Costa Rica prohibiendo la fecundación in vitro en dicho país. Exp: 95-001734-0007-CO. Res: 2000-02306 


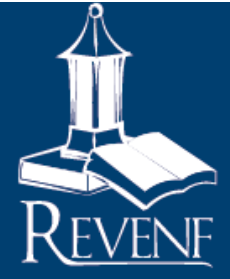

Revista Electrónica Enfermeria Actual en costa Rica

Urries, A. (2010). Seleccionando el mejor embrión. Recuperado de

http://www.hvn.es/servicios asistenciales/ginecologia y obstetricia/ficheros/curso2010 reprod 12 seleccionando_al mejo $\underline{\text { r embrion aurries.pdf }}$ 